\title{
O RECURSO MAIS IMPORTANTE PARA AS ORGANIZAÇÕES SÃO MESMO AS PESSOAS? UMA ANÁLIS̉E DA PRODUÇÃO CIENTIIFICA SOBRE QUALIDADE DE VIDA NO TRABALHO (QVT)
}

\author{
PEOPLE ARE REALLY THE MOST IMPORTANT \\ RESOURCE OF ORGANIZATIONS? AN ANALYSIS OF \\ THE SCIENTIFIC PRODUCTION ON \\ QUALITY OF WORKING LIFE (QWL)
}

Recebido 28/06/2012

Aceito $08 / 07 / 2012$

Aline Botelho Schneider Venson ${ }^{1}$, Gabriela Gonçalves Silveira Fiates ${ }^{2}$ Ademar Dutra ${ }^{3}$, Marcelo Lopes Carneiro ${ }^{4}$, Cristina Martins ${ }^{5}$

\begin{abstract}
RESUMO
O discurso nas organizações em geral, independente de porte ou natureza, aborda recorrentemente a relevância do capital humano para o sucesso e a relação direta entre o desempenho dos colaboradores e seus resultados. Estudos sobre a qualidade de vida no trabalho (QVT) são de extrema importância para entender as variáveis que têm impacto sobre a performance e os resultados, tanto da organização, quanto de seus colaboradores. Para avaliar o quanto as pesquisas científicas refletem essa importância, neste estudo tem-se como objetivo analisar a produção científica acerca do tema QVT no Brasil, observando, para isso, os artigos publicados nos anais do Encontro da Associação Nacional de Pós-Graduação e Pesquisa em Administração (EnANPAD). Trata-se de uma pesquisa bibliométrica da produção acadêmica do período de 1997 a2011. Após a coleta das informações, foram encontrados 64artigos que atendiam a pelo menos uma das palavras-chave definidas pelo escopo deste estudo. Como resultado verificou-se que o tema QVT, até 2008, ainda era abordado na produção científica de forma incipiente, com cunho investigativo, com o intuito apenas de diagnosticar índices de QVT, sem buscar identificar seus reflexos na organização como um todo. No entanto, a partir de 2009, já se pode perceber certo amadurecimento quanto à identificação de trabalhos que buscaram avaliar as relações entre QVT, responsabilidade social, comprometimento organizacional e, inclusive, desempenho.
\end{abstract}

Palavras-chave: Qualidade de Vida no Trabalho; bibliometria; produção científica.

\footnotetext{
${ }^{1}$ Possui graduação em Administração com habilitação em Comércio Exterior pela Universidade do Vale do Itajaí - UNIVALI e mestrado em Administração pela Universidade do Sul de Santa Catarina - UNISUL. Tubarão, Santa Catarina, Brasil. E-mail: alinebotelhos@ hotmail.com

${ }^{2}$ Possui graduação em Engenharia Mecânica pela Universidade Federal de Santa Catarina - UFSC, mestrado em Engenharia de Produção pela UFSC e Doutorado em Engenharia de Produção pela UFSC. Professora do Departamento de Administração da UFSC. Florianópolis, Santa Catarina, Brasil. E-mail: gabriela.fiates@ufsc.br

${ }^{3}$ Possui graduação em Administração pela Universidade Federal de Santa Catarina - UFSC, mestrado em Engenharia de Produção pela UFSC, doutorado em Engenharia de Produção pela UFSC. Professor e Pesquisador da Universidade do Sul de Santa Catarina - UNISUL. Tubarão, Santa Catarina, Brasil. E-mail: ademar.unisul@gmail.com

${ }^{4}$ Possui graduação em Administração pela Universidade Federal de Santa Catarina - UFSC e mestrado em Administração pela Universidade do Sul de Santa Catarina - UNISUL. Tubarão, Santa Catarina, Brasil. E-mail: marcelozyx@hotmail.com

${ }^{5}$ Possui graduada em Sistemas de Informação pela Universidade do Sul de Santa Catarina - UNISUL, mestrado em Administração pela Universidade do Sul de Santa Catarina - UNISUL. Doutoranda em Administração pela Universidade Federal de Santa Catarina - UFSC. E-mail: crismartins2611@gmail.com
} 


\section{ABSTRACT}

The discourse in organizations in general, regardless of size or nature, focuses on a recurring basis the relevance of human capital for success and direct relationship between employee performance and organizational results. Studies about quality of work life are extremely important to understand the variables that impact performance and results for both: the organization and its employees. To evaluate how scientific research reflect this importance, this study has been developed to analyze the scientific production on the subject of quality of work life - QWL in Brazil, analyzing the articles published in the annals of EnANPAD - Meeting of the National Association Postgraduate and Research in Administration. This is a bibliometric research of academic production in the period 1997 to 2011 . After collection of information were found 64 articles that met at least one of keywords defined by the scope of this study. As a result it was found that the subject of quality of working life until 2008 was still discussed in scientific literature only incidentally, with an investigative nature, in order to diagnose only QVT index, without seeking to identify its impact on the organization as a whole. However, from 2009 it can already notice a maturity based on the identification of works that investigated the relationship between $Q W L$, social responsibility, organizational commitment and, including, performance.

Keywords: Quality of Working Life; bibliometrics; scientific production.

\section{INTRODUÇÃO}

O mundo do trabalho vem passando por profundas transformações. Temáticas como a globalização, a flexibilização, a competitividade e até a planificação do mundo, proposta por Friedman (2005), surgem para explicar o dinamismo e a complexidade dos fenômenos que acontecem atualmente na sociedade e, consequentemente, nas organizações. Com isso, novas formas de organização do trabalho são objeto de análises daqueles que atuam nas ou estudam as organizações, bem como os fatores que propiciam às empresas fontes de vantagem competitiva.

Frente ao cenário organizacional de constantes e fortes mudanças de estruturas e de gestão, latente exigência de agilidade e produtividade atreladas a produtos e serviços com altos padrões de qualidade e competitividade, o fator humano aparenta ser o elemento diferenciador das organizações, o que requer atenção especial por parte das empresas. Nesse sentido, estudos referentes à qualidade de vida no trabalho (QVT) vêm sendo postos em evidência devido à atual importância dada ao capital humano como fator de sobrevivência e de melhoria da competitividade empresarial. Compreender que ações de QVT são necessárias para atingir a lucratividade máxima, é determinante para o sucesso das organizações.

No contexto atual, autores defendem que um programa de QVT é de fundamental importância para a sobrevivência das empresas, haja vista que o mercado altamente competitivo requer habilidades, conhecimentos e energias humanas capazes de destacarem-se em relação aos demais concorrentes (MENDES; OLIVEIRA, 1998). No entanto, segundo a Organização Mundial da Saúde (OMS, 2003), a qualidade de vida lida com o que as pessoas "sentem" sobre sua condição de saúde ou suas consequências, tratando-se, dessa forma, de um construto de "bem-estar subjetivo".

No centro das organizações e, por conseguinte, de todo processo de mudanças contínuas, está o indivíduo, que, embora esteja inquestionavelmente envolvido com a organização, está ao mesmo tempo cada vez mais alheio aos impactos que vem sofrendo. Caso não haja um bom diagnóstico, um planejamento e uma implementação de ações para a promoção da QVT, os resultados organizacionais podem ser afetados de forma significativa. Um indivíduo, quando percebe que seus anseios não estão sendo atingidos, sente-se inseguro e desmotivado, e por consequência, tende a não produzir com o grau de interesse e comprometimento que a empresa espera.

Tolfo e Piccinini (2001) enfatizam que, embora a temática da QVT tenha recebido 
considerável atenção a partir da década de 80, ainda existe alguma incerteza com relação ao quanto a QVT afeta os resultados econômico-financeiros das organizações. Muitas são as pesquisas referentes à QVT, mas, em sua maioria, são sempre realizadas com foco voltado ao âmbito humano da questão, ao lado intrínseco do desempenho. Contudo, a QVT pode ser abordada de forma muito mais ampla que como um simples modismo ou plano de benefícios dados aos colaboradores pela organização. Ela também pode ser vista como um fator diretamente ligado ao desempenho organizacional, pois indivíduos inseridos em um ambiente onde a QVT é elevada tendem a oferecer à organização melhores resultados, o que, consequentemente, se reflete no desempenho organizacional como um todo.

Dada a importância da QVT para as organizações, buscou-se desenvolver este estudo para responder a seguinte pergunta de pesquisa: Qual o foco da QVT na produção científica brasileira do período compreendido entre 1997 a 2011? Para alcançar esse objetivo, analisou-se a produção científica acerca desse tema nos anais do Encontro da Associação Nacional de PósGraduação e Pesquisa em Administração (EnANPAD), por considerar-se que esse evento integra o maior número de pesquisas desenvolvidos nos programas brasileiros de pós-graduação em Administração.

A relevância deste estudo está fundamentada na importância que a QVT passa a ter ao longo dos anos no cenário das organizações, sobretudo nas últimas décadas, já que estas entendem o indivíduo como uma importante fonte de resultados, e, ainda, na relevância de uma comparação entre a realidade das empresas e as pesquisas acadêmicas que tratam dessa temática. Acredita-se também que embora seja inegável a importância do tema, ainda se pode perceber que as pesquisas na área parecem incipientes. Barreto et al. (2011) pesquisaram os temas emergentes em Gestão de Pessoas e, embora este não tenha sido um tema relacionado na pesquisa como emergente, esperava-se encontrar um número mais expressivo de artigos que tratassem da qualidade de vida no trabalho. No entanto, dos 121 artigos selecionados para a pesquisa, apenas três trouxeram preocupação com este tópico.

Com isso acredita-se que este trabalho seja útil para pesquisadores e pessoas que busquem conhecer e se aprofundar no tema QVT, oferecendo uma base de artigos analisados e devidamente classificados, além de mostrar as tendências e demonstrar as dimensões mais presentes na produção científica em questão.

O presente artigo está estruturado da seguinte forma: além desta seção de caráter introdutório, a seção dois - Pesquisas Anteriores- aborda sucintamente a produção científica acerca do tópico investigado nesta pesquisa, relatando estudos anteriormente realizados, além de uma introdução ao tema QVT; na seção três - Procedimentos Metodológicos -o método utilizado será exposto; a seção quatro - Apresentação e Análise dos Resultados - apresenta os resultados alcançados na investigação da produção científica acerca do tema QVT e, na seção cinco - Considerações Finais -, são apresentadas as reflexões e recomendações para futuras pesquisas. Por fim, a seção seis - Referências Bibliográficas -, apresenta a bibliografia utilizada na pesquisa.

\section{PESQUISAS ANTERIORES}

A análise da produção científica brasileira iniciou-se com o estudo de Machado-da-Silva, Cunha e Amboni (1990), que buscaram, por meio da análise de artigos da área de organizações, publicados entre os anos de 1985 e 1989, demonstrar a necessidade de avaliar a produção científica brasileira, com o objetivo de identificar seus pontos fortes e fracos. Desde então, as pesquisas que visam a análise da produção científica brasileira têm sido, cada vez mais, utilizadas como instrumento 
para verificação das características dos estudos publicados e possuem principalmente duas vertentes: (a) as que analisam a administração de forma ampla; (b) as que analisam cada disciplina, ou tema específico, isoladamente (ROSSONI; FERREIRA JÚNIOR; HOCAYEN-DA-SILVA, 2006).

Pode-secitar diversos estudos que visaram analisar a produção científica da Administração de forma ampla, como os de Bertero, Caldas e Wood Jr. (1998, 1999), Fleury (2003) e, ainda, Wood Jr. e Paula (2002). Entre os trabalhos que enfocam a análise de temas isoladamente, podese citar os trabalhos de Serra, Pereira e Oliveira (2007), em que é analisada a produção em torno da teoria de Resource-based-view (RBV), e Schneider, Carneiro e Dutra (2009), que analisaram a produção científica brasileira sobre avaliação de desempenho.

Nos meta estudos citados, cada campo de estudo que é foco de pesquisa recebe um tratamento específico no que diz respeito aos dados analisados. No caso do artigo de Serra, Ferreira, Pereira e Lissoni (2008), foram analisadas as inter-relações dos artigos com as teorias de autores que influenciaram as pesquisas, e, também, as tendências das metodologias dos artigos. Já no estudo de Medeiros e Oliveira (2007), foram analisadas, de maneira aprofundada, a tipologia dos métodos e a corrente científica dos artigos.

Também se pode citar alguns outros meta estudos já publicados nos anais do EnANPAD, como forma de confirmar a tendência de analisar a produção científica como verificação do estado da arte de determinado assunto, como o estudo de Cappelle et al. (2006), que analisou a inserção de uma visão de gênero na produção científica e o estudo de Closs, Arambutu e Antunes (2006), que analisou a produção científica sobre o ensino de Administração, e chegaram à conclusão de que os artigos apontaram uma necessidade de mudança no contexto e na forma do ensino de Administração no Brasil.

Em relação ao tema QVT, pode-se citar diversos trabalhos, mas raros focados na análise da produção científica acerca do tema. Geralmente, pesquisas ligadas a QVT apresentam cunho investigatório, em que o objetivo tem sido identificar/avaliar a QVT em determinado caso. Já como pesquisa voltada à produção científica sobre QVT, pode-se citar o trabalho de Medeiros e Oliveira (2007), que focou a produção científica sobre o tema nos anais do EnANPAD, no período de 2001 a 2005. O trabalho analisou as seguintes variáveis: (i) base epistemológica; (ii) base teórica; (iii) base morfológica. Em seguida, buscou (iv) traçar um perfil metodológico; para, depois, (v) levantar um padrão de referência; e a (vi) demografia de autoria. Dantas, Sawada e Malerbo (2003) realizaram uma pesquisa sobre qualidade de vida, na qual revisaram a produção científica sobre o tema nas universidades públicas do Estado de São Paulo. Frank Pot, em seu trabalho de pesquisa do ano de 2003, "Productivity and utilisation of the potential work force", apresenta a preocupação de vários países em equalizar os procedimentos de qualidade de vida dos trabalhadores, correlacionando a QVT com a produtividade, mostrando o quanto as empresas deixam de ganhar, o quanto gastam e, além disso, o custo social da má gestão dos processos que afetam a saúde dos trabalhadores (POT, 2003).

Nesse contexto, a presente pesquisa visa continuar tais estudos, buscando aprofundar o conhecimento sobre a realidade da produção científica sobre QVT no Brasil.

\section{QUALIDADE DE VIDA NO TRABALHO (QVT)}

As organizações, a partir da década de 80, passaram a concorrer no mercado de forma mais intensa, devido ao processo de globalização da economia, a o contínuo avanço da tecnologia e à consequente compressão do tempo e do espaço. A qualidade de vida no trabalho (QVT), tema originalmente de competência da área de Gestão de Pessoas - tem sido ampliado, de forma a ser inserida no âmbito das estratégias organizacionais, cobrindo os níveis tático e operacional, 
embora, às vezes, o tema ainda seja tratado como modismo. O referido tema ganhou relevância na era atual e vem conquistando espaço nas organizações nacionais e internacionais, além de ter se tornado objeto de estudo de diversas pesquisas.

Segundo Sampaio (2004), a expressão QVT apareceu na literatura especializada no início da década de 1950. Cardoso (1999) destaca, com propriedade, um histórico acerca do tema. Porém, a preocupação com a QVT por parte das organizações teve início a partir das convenções da Organização Internacional do Trabalho (OIT), em que os países membros adequaram a legislação de seus países visando a melhoria do trabalho, principalmente no que concerne à saúde, higiene e segurança do trabalhador.

De acordo com Albuquerque e Limongi-França (1998), QVT refere-se a um conjunto de ações de uma empresa que envolve diagnóstico e implantação de melhorias e inovações gerenciais, tecnológicas e estruturais, dentro e fora do ambiente de trabalho, visando propiciar condições plenas de desenvolvimento humano para e durante a realização do trabalho.

No entanto, o tema QVT apresenta um caráter mais amplo, contemplando aspectos físicos e emocionais (JARDIM; BARRETO; ASSUNÇÃO, 2007). Dessa forma, esse tema deveria receber uma atenção multidisciplinar, por exemplo: (i)Administração: gerenciamento de recursos para obter melhores resultados para as organizações; (ii) Economia: distribuição equilibrada de recursos, bens e serviços; (iii) Saúde: preservação da integridade física, mental e social do indivíduo; (iv) Ergonomia: maior conforto para a execução do trabalho; (v) Sociologia: relações de quem influencia e é influenciado dentro da organização; (vi) Engenharia: uso da tecnologia, organização do trabalho e controle dos processos; (vii)Psicologia: perspectivas e significados individuais sobre o trabalho e (viii) Ecologia: inserção do homem no ecossistema e consideração do mesmo como responsável pela preservação da natureza e dos seres vivos.

Segundo Fernandes (1996), o termo qualidade de vida lembra, apenas, ideias associadas a melhorias nas condições físicas, menor jornada de trabalho e maiores salários. Contudo, o enfoque principal é a reformulação da relação do trabalho em si com o empregado, gerando mais eficácia, produtividade e satisfação das necessidades básicas dos empregados. Para LimongiFrança (2003), os programas de Qualidade Total só apresentarão resultados máximos quando passarem a dar mais valor à voz do cliente interno, em relação a suas necessidades e expectativas, culminando em maior desenvolvimento de seu potencial, viabilizando maior qualidade de produtos e serviços, atendendo aos desejos dos clientes e criando vantagem competitiva para a organização. A QVT só faz sentido quando deixa de ser restrita a programas internos de saúde ou lazer e passa a ser discutida num sentido mais amplo, incluindo a qualidade das relações de trabalho e suas consequências na saúde das pessoas e da organização.

Para Nadler e Lawler (1983) apud Rodrigues (1999), QVT é a grande esperança das organizações para atingirem altos níveis de produtividade, sem esquecer a motivação e satisfação do indivíduo. A ênfase da QVT está no individuo, de forma que o capital intelectual, isto é, o conhecimento compartilhado de cada um, se torna o ativo mais importante da empresa, objetivando o aumento da lucratividade. Por esse motivo, o estudo da QVT em empresas tem lugar de destaque, independentemente de o cenário econômico mostrar recessão ou crescimento, perda de poder aquisitivo ou aumento do desemprego.

Para demonstrar os fatores que afetam a QVT, foram desenvolvidos modelos de indicadores de qualidade de vida por diversos autores ao longo dos anos. Esses modelos vêm sendo utilizados como guias para o diagnóstico da qualidade de vida nas organizações. Dentre eles, pode-se citar o modelo de Walton (1973), Hackman e Oldhan (1975), Westley (1979), Nadler e Lawer (1983), Werther e Davis (1983), Huse e Cummings (1985), Cooper et al. (1988), 
Marques (1993), Fernandes (1996), entre outros. Os vários modelos teóricos sobre QVT vêm sendo reformulados e combinados de forma a adaptarem-se aos novos contextos, uma vez que a percepção das dimensões da QVT pode se apresentar de maneira diferente para os indivíduos e para as organizações.

Nesse sentido, a partir dos modelos supracitados, a QVT envolve uma constelação de fatores: (i) a satisfação com o trabalho executado; (ii) as possibilidades futuras na organização; (iii) o reconhecimento pelos resultados alcançados; (iv) o salário recebido; (v) os benefícios auferidos; (vi) o relacionamento humano dentro do grupo e da organização; (vii) o ambiente psicológico e físico de trabalho; (viii) a liberdade e responsabilidade de decisão; (ix) as possibilidades de participação. De fato, vários trabalhos têm buscado relacionar tais fatores à percepção de valor do cliente interno - colaborador - e seus efeitos sobre a própria organização. Gheno e Berlitz (2011, p. 285), por exemplo, ao pesquisarem colaboradores do nível operacional de uma empresa multinacional da região de Porto Alegre, concluíram que "de um modo geral, os colaboradores entrevistados consideram que os programas de remuneração estratégica e benefícios oferecidos pela empresa incentivam o seu desempenho em busca de melhores resultados". Rosa e Ibdaiwi (2012) também encontraram resultados interessantes ao buscar identificar as causas do turn over de uma empresa de fast food de Santa Maria-RS, apontando, por exemplo, como uma das causas a desmotivação dos colaboradores ao sentirem-se desvalorizados e não reconhecidos pelo seu trabalho e esforço extra. Os autores encontraram ainda como causa do turn over a "insalubridade" emocional e psicológica do ambiente dessa organização, uma vez que os colaboradores convivem com a pressão da política empresarial a que são submetidos.

Sendo assim, a QVT contempla os aspectos intrínsecos (conteúdo) e extrínsecos (contextos) do cargo ocupado pelo individuo, afetando as atitudes pessoais e os comportamentos relevantes para a produtividade individual e grupal, tais como: motivação para o trabalho, adaptabilidade a mudanças no ambiente de trabalho, criatividade e vontade de inovar ou aceitar mudança.

\section{PROCEDIMENTOS METODOLÓGICOS}

Esta seção apresenta as características gerais da pesquisa, bem como o método utilizado, a descrição detalhada dos procedimentos e, por fim, apresenta a forma como os resultados foram analisados e classificados.

\subsection{Características da Pesquisa}

O presente estudo possui um caráter descritivo e constitui-se como uma pesquisa bibliográfica, pois é desenvolvido a partir de material já elaborado, constituído de artigos científicos já publicados nos anais do Encontro da Associação Nacional de Pós-Graduação e Pesquisa em Administração (EnANPAD), visando apontar as características e tendências da produção científica Brasileira na área de qualidade de vida no trabalho (QVT), no período de 1997 a 2011.

\subsection{Método de Pesquisa}

O método, segundo Kuhnen (2004, p.46), pode ser definido como sendo os procedimentos adotados pelo pesquisador ou o caminho que este estabelece para realizar a pesquisa. No caso deste estudo, buscou-se analisar a incidência de artigos relacionados à QVT. Para tanto, foram 
seguidos os seguintes procedimentos:

i. Seleção dos anais de congresso a serem utilizados como fontes para coleta de dados - os Anais do EnANPAD - por ser este o congresso de maior representatividade na área de pesquisa, no âmbito nacional;

ii. Determinação do período de tempo a ser coberto pela investigação - 1997 a 2011 - pois um tempo mais longo levaria a resultados mais abrangentes;

iii. Determinação das palavras-chaves a serem usadas na busca bibliográfica-qualidade de vida no trabalho (QVT);

iv. Realização da busca com base no período e nas palavras-chaves determinados em (ii) e (iii), no site do evento;

v. Refinamento dos artigos encontrados, pois alguns dos resultados não se encaixavam no escopo deste estudo;

Exclusão de artigos com defeito ou corrompidos da amostra.

4.3 Classificação dos resultados

Os artigos foram classificados da seguinte forma:

i. Quanto aos seus autores - descritos os nomes completos dos seus autores;

ii. Quanto à instituição de vinculação dos autores - descritas, preferencialmente, a instituição de vinculação do trabalho, quando essa não era explícita foi pesquisada na Plataforma Lattes do Ministério da Educação (MEC), quando essa não existia, foi classificada como não identificada/encontrada;

iii. Quanto à abordagem metodológica - separados em artigos teóricos, empíricos ou estudos de caso, conforme a literatura corrente, como por exemplo a classificação de Phelan, Ferreira e Salvador (2002);

iv. Quanto ao enquadramento metodológico da pesquisa - tipo e natureza da pesquisa, estratégia utilizada e técnica utilizada para coleta e análise dos dados;

v. Quanto ao modelo de análise de QVT - caracterizados conforme o modelo teórico utilizado para embasar a pesquisa, quando não estava explícito o modelo utilizado, ou quando o autor elaborava um modelo, foram classificados como modelo próprio (MP) e não informado (NI);

vi. Quanto à esfera organizacional - divididos nas esferas pública, privada e não identificável, quando não era descrita e nem implícita no conteúdo do estudo;

vii. Ano e área de publicação - de acordo com o ano da publicação do artigo e área publicada no evento;

viii. Referência mais utilizada - tabuladas todas as referências utilizadas para a elaboração dos 57 artigos, a fim de identificar quais obras serviram mais comumente de base para a formação de um referencial teórico sobre QVT. 


\section{APRESENTAÇÃO E ANÁLISE DOS DADOS}

Dentre os artigos selecionados para este estudo, pode-se afirmar que a produção científica brasileira de Administração triplicou no período estudado. Levando em conta o ano de 1997, primeiro ano do levantamento, e o ano de 2008, percebe-se que a produção científica de Administração cresceu aproximadamente $400 \%$ na base selecionada. No entanto, após 2008, o número de artigos apresentados ao evento e, em específico sobre qualidade de vida no trabalho (QVT), diminuíram. É possível averiguar todos os detalhes dessa evolução cronológica demonstrada na Tabela 1. Também, através da Tabela 1, pode-se notar o grande número de publicações em todo o período analisado, cerca de 9762 artigos, além do número de artigos selecionados dentre o escopo desta pesquisa, apresentado na seção da metodologia, que perfaz o total de 64artigos.

Tabela 1: Produção acadêmica geral e sobre QVT do Encontro da Associação Nacional de Pós-Graduação e Pesquisa em Administração (EnANPAD).

\begin{tabular}{lcccccccccccccccc}
\hline $\begin{array}{c}\text { Veículo / } \\
\text { Período }\end{array}$ & $\mathbf{9 7}$ & $\mathbf{9 8}$ & $\mathbf{9 9}$ & $\mathbf{0 0}$ & $\mathbf{0 1}$ & $\mathbf{0 2}$ & $\mathbf{0 3}$ & $\mathbf{0 4}$ & $\mathbf{0 5}$ & $\mathbf{0 6}$ & $\mathbf{0 7}$ & $\mathbf{0 8}$ & $\mathbf{0 9}$ & $\mathbf{1 0}$ & $\mathbf{1 1}$ & TOTAL \\
\hline TODOS EnANPAD & 249 & 250 & 270 & 364 & 426 & 554 & 630 & $\mathbf{7 9 9}$ & $\mathbf{7 8 7}$ & $\mathbf{8 3 6}$ & 964 & 1005 & 906 & 842 & 880 & $\mathbf{9 7 6 2}$ \\
QVT NO EnANPAD & 2 & 2 & 7 & 3 & 4 & 11 & 7 & 4 & 6 & 2 & 2 & 7 & 3 & 2 & 2 & $\mathbf{6 4}$ \\
$\begin{array}{l}\text { \% ARTIGOS QVT NO } \\
\text { EnANPAD }\end{array}$ & 0,8 & 0,8 & 2,6 & 0,8 & 0,9 & 2,0 & 1,1 & 0,5 & 0,8 & 0,2 & 0,2 & 0,7 & 0,3 & 0,2 & 0,2 & $\mathbf{0 , 7}$ \\
\hline
\end{tabular}

Fonte: Elaborado pelos autores.

Percebe-se que, comparando o total de artigos que tratam sobre QVT ao total de artigos publicados anualmente no evento, como mostra a Tabela 1, o número daquele é pequeno em relação a este. Na amostra analisada, pode-se verificar que o volume não consegue ultrapassar sequer $3 \%$ do total de artigos em nenhum dos anos, o que é um valor ainda irrisório, considerandose a importância do tema nas organizações.

Iniciando uma classificação dos artigos que compõe a amostra, foi possível verificar que não existe predominância no tipo de abordagem metodológica utilizada. O que se pode inferir é que, dentre a amostra utilizada, é mínimo o número de pesquisas teóricas sobre o tema.

Tabela 2 - Produção acadêmica de QVT por abordagem metodológica e ano.

\begin{tabular}{ccccccccccccccccc}
$\begin{array}{c}\text { Abordagem / } \\
\text { Período }\end{array}$ & $\mathbf{9 7}$ & $\mathbf{9 8}$ & $\mathbf{9 9}$ & $\mathbf{0 0}$ & $\mathbf{0 1}$ & $\mathbf{0 2}$ & $\mathbf{0 3}$ & $\mathbf{0 4}$ & $\mathbf{0 5}$ & $\mathbf{0 6}$ & $\mathbf{0 7}$ & $\mathbf{0 8}$ & $\mathbf{0 9}$ & $\mathbf{1 0}$ & $\mathbf{1 1}$ & TOTAL \\
\hline Estudo de Caso & 1 & 1 & 5 & 2 & 3 & 5 & 2 & 1 & 3 & 0 & 2 & 2 & 1 & 1 & 1 & $\mathbf{2 8}$ \\
Teórico & 0 & 0 & 0 & 0 & 0 & 0 & 0 & 0 & 1 & 0 & 0 & 0 & 0 & 0 & 0 & $\mathbf{1}$ \\
Empírico & 1 & 1 & 2 & 1 & 1 & 6 & 5 & 3 & 2 & 2 & 0 & 5 & 2 & 1 & 1 & $\mathbf{3 3}$ \\
TOTAL & $\mathbf{2}$ & $\mathbf{2}$ & $\mathbf{7}$ & $\mathbf{3}$ & $\mathbf{4}$ & $\mathbf{1 1}$ & $\mathbf{7}$ & $\mathbf{4}$ & $\mathbf{6}$ & $\mathbf{2}$ & $\mathbf{2}$ & $\mathbf{7}$ & $\mathbf{3}$ & $\mathbf{2}$ & $\mathbf{2}$ & $\mathbf{6 4}$ \\
\hline
\end{tabular}

Fonte: Elaborado pelos autores.

Tanto nos estudos de caso, quanto nas pesquisas empíricas encontradas é predominante o emprego do tema como forma de diagnóstico, seja ele em uma única empresa, seja entre um universo mais abrangente. Poucos são os casos em que a pesquisa, ao invés de avaliar a QVT 
de uma determinada população, busca relacioná-la com outros fatores organizacionais, como desempenho, motivação, responsabilidade social, dentre outros. Essas relações começaram a aparecer nos artigos a partir de 2009, com um artigo relacionando QVT com comprometimento organizacional e outro relacionando QVT com responsabilidade social da empresa. Em 2010, aparece novamente o tema relacionado ao comprometimento e, em 2011, aparece o primeiro artigo que busca analisar a relação entre QVT e desempenho organizacional.

Dentre os artigos da amostra estudada, pode-se agrupá-los quanto à área de publicação no evento. Lembrando que as áreas do EnANPAD estão assim distribuídas: Administração Pública e Gestão Social (APS), Estudos Organizacionais (EOR), Gestão de Operações e Logística (GOL), Gestão de Pessoas e Relações de Trabalho (GRP) e Marketing (MKT).

Por meio dessa análise, infere-se a área que mais aborda a QVT, conforme mostra a Tabela 3.

Tabela 3 - Produção acadêmica de QVT por área do EnANPAD e ano.

\begin{tabular}{ccccccccccccccccc}
\hline $\begin{array}{c}\text { Área/ } \\
\text { Período }\end{array}$ & $\mathbf{9 7}$ & $\mathbf{9 8}$ & $\mathbf{9 9}$ & $\mathbf{0 0}$ & $\mathbf{0 1}$ & $\mathbf{0 2}$ & $\mathbf{0 3}$ & $\mathbf{0 4}$ & $\mathbf{0 5}$ & $\mathbf{0 6}$ & $\mathbf{0 7}$ & $\mathbf{0 8}$ & $\mathbf{0 9}$ & $\mathbf{1 0}$ & $\mathbf{1 1}$ & TOTAL \\
APS & 0 & 0 & 0 & 0 & 0 & 0 & 0 & 1 & 1 & 0 & 0 & 0 & 0 & 0 & 0 & $\mathbf{2}$ \\
EOR & 0 & 0 & 2 & 1 & 2 & 5 & 6 & 1 & 2 & 0 & 1 & 1 & 0 & 1 & 0 & $\mathbf{2 2}$ \\
GOL & 0 & 0 & 0 & 0 & 1 & 0 & 0 & 0 & 0 & 0 & 0 & 0 & 0 & 0 & 0 & $\mathbf{1}$ \\
GRP & 2 & 2 & 5 & 2 & 1 & 6 & 1 & 1 & 3 & 2 & 1 & 6 & 3 & 1 & 2 & $\mathbf{3 8}$ \\
MKT & 0 & 0 & 0 & 0 & 0 & 0 & 0 & 1 & 0 & 0 & 0 & 0 & 0 & 0 & 0 & $\mathbf{1}$ \\
TOTAL & $\mathbf{2}$ & $\mathbf{2}$ & $\mathbf{7}$ & $\mathbf{3}$ & $\mathbf{4}$ & $\mathbf{1 1}$ & $\mathbf{7}$ & $\mathbf{4}$ & $\mathbf{6}$ & $\mathbf{2}$ & $\mathbf{2}$ & $\mathbf{7}$ & $\mathbf{3}$ & $\mathbf{2}$ & $\mathbf{2}$ & $\mathbf{6 4}$ \\
\hline
\end{tabular}

Fonte: Elaborado pelos autores.

Por meio da tabela 3, pode-se verificar que a produção científica sobre QVT aparece principalmente nas áreas de gestão de pessoas e estudos organizacionais, o que é justificável, visto que aspectos ligados aos trabalhadores são realmente analisados pelo âmbito humano e comportamental, e, mais raramente, relacionados com outras temáticas, como, por exemplo, opção estratégica.

Também foram analisadas as esferas organizacionais às quais os artigos se referiam em seus estudos.

Tabela 4 - Produção acadêmica de QVT por esfera organizacional e ano.

\begin{tabular}{ccccccccccccccccc}
\hline Esf. / Período & $\mathbf{9 7}$ & $\mathbf{9 8}$ & $\mathbf{9 9}$ & $\mathbf{0 0}$ & $\mathbf{0 1}$ & $\mathbf{0 2}$ & $\mathbf{0 3}$ & $\mathbf{0 4}$ & $\mathbf{0 5}$ & $\mathbf{0 6}$ & $\mathbf{0 7}$ & $\mathbf{0 8}$ & $\mathbf{0 9}$ & $\mathbf{1 0}$ & $\mathbf{1 1}$ & TOTAL \\
\hline Privada & 1 & 1 & 4 & 2 & 2 & 6 & 6 & 3 & 2 & 2 & 1 & 3 & 1 & 2 & 1 & $\mathbf{3 7}$ \\
Pública & 0 & 0 & 2 & 1 & 2 & 1 & 0 & 0 & 3 & 0 & 1 & 3 & 1 & 0 & 0 & $\mathbf{1 4}$ \\
Ambos & 0 & 1 & 1 & 0 & 0 & 4 & 0 & 1 & 0 & 0 & 0 & 0 & 0 & 0 & 0 & $\mathbf{7}$ \\
Não & 1 & & & & & & 1 & & 1 & & & 1 & 1 & 0 & 1 & $\mathbf{6}$ \\
identificável & 1 & & & & & & $\mathbf{7}$ & $\mathbf{4}$ & $\mathbf{6}$ & $\mathbf{2}$ & $\mathbf{2}$ & $\mathbf{7}$ & $\mathbf{3}$ & $\mathbf{2}$ & $\mathbf{2}$ & $\mathbf{6 4}$ \\
TOTAL P/ ANO & $\mathbf{2}$ & $\mathbf{2}$ & $\mathbf{7}$ & $\mathbf{3}$ & $\mathbf{4}$ & $\mathbf{1 1}$ & $\mathbf{7}$ & $\mathbf{4}$
\end{tabular}

Fonte: Elaborado pelos autores.

Um aspecto importante, também averiguado, foi a natureza da abordagem utilizada pelos autores para delinear sua pesquisa, como mostra o Gráfico1. 
Gráfico1: Abordagem utilizada.

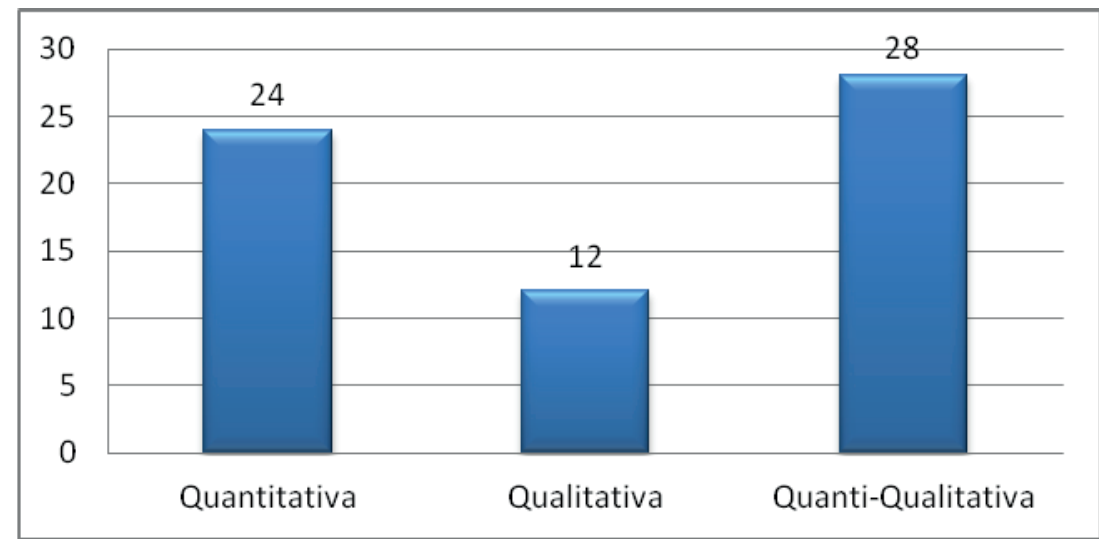

Fonte: Elaborado pelos autores.

Pode-se verificar a predominância de uma abordagem quanti-qualitativa, fato que pode ser explicado pela própria característica do tema QVT, que trabalha aspectos tanto aspectos quantificáveis quanto aspectos substanciais ligados ao individuo na organização. As análises quantitativas aparecem geralmente para comprovar de uma forma positivista as questões ligadas à QVT e diagnosticar o índice de QVT em determinada organização, e são corroboradas por análises qualitativas, nas quais geralmente se encontram depoimentos e discursos dos pesquisados.

Também pode ser observado o tipo de pesquisa utilizado nos artigos, conforme mostra a Tabela 5.

Tabela 5 - Produção de QVT por tipo de pesquisa e ano.

\begin{tabular}{ccccccccccccccccc}
\hline $\begin{array}{c}\text { Tipo Pesquisa / } \\
\text { Período }\end{array}$ & $\mathbf{9 7}$ & $\mathbf{9 8}$ & $\mathbf{9 9}$ & $\mathbf{0 0}$ & $\mathbf{0 1}$ & $\mathbf{0 2}$ & $\mathbf{0 3}$ & $\mathbf{0 4}$ & $\mathbf{0 5}$ & $\mathbf{0 6}$ & $\mathbf{0 7}$ & $\mathbf{0 8}$ & $\mathbf{0 9}$ & $\mathbf{1 0}$ & $\mathbf{1 1}$ & TOTAL \\
\hline Exploratória & 0 & 1 & 3 & 0 & 0 & 4 & 4 & 2 & 0 & 0 & 0 & 1 & 0 & 0 & 1 & $\mathbf{1 6}$ \\
Descritiva & 1 & 0 & 1 & 1 & 1 & 0 & 3 & 0 & 3 & 1 & 1 & 3 & 1 & 2 & 1 & $\mathbf{1 9}$ \\
Bibliográfica & 0 & 0 & 0 & 0 & 0 & 0 & 0 & 0 & 1 & 0 & 0 & 0 & 0 & 0 & 0 & $\mathbf{1}$ \\
Descritiva- & 1 & 1 & 1 & 1 & 0 & 4 & 0 & 1 & 2 & 1 & 0 & 3 & 1 & 0 & 0 & $\mathbf{1 6}$ \\
exploratória & 0 & 0 & 2 & 1 & 3 & 3 & 0 & 1 & 0 & 0 & 1 & 0 & 1 & 0 & 0 & $\mathbf{1 2}$ \\
Outras & $\mathbf{2}$ & $\mathbf{2}$ & $\mathbf{7}$ & $\mathbf{3}$ & $\mathbf{4}$ & $\mathbf{1 1}$ & $\mathbf{7}$ & $\mathbf{4}$ & $\mathbf{6}$ & $\mathbf{2}$ & $\mathbf{2}$ & $\mathbf{7}$ & $\mathbf{3}$ & $\mathbf{2}$ & $\mathbf{2}$ & $\mathbf{6 4}$ \\
\hline TOTAL & $\mathbf{2}$ &
\end{tabular}

Fonte: Elaborado pelos autores.

Com base na amostra pesquisada, pode-se inferir que na produção científica referente à QVT existe uma predominância de pesquisas descritivas e/ou exploratórias. Como a maioria dos artigos busca investigar ou diagnosticar o estágio de implementação de ações promotoras de qualidade de vida presentes na organização, é comum que tais tipos de pesquisa tenham sido adotados, embora, em uma análise dos artigos, pode-se perceber que muitos dos apresentados como exploratórios não se configuravam como tal, uma vez que tratavam de um tema já amplamente discutido na literatura.

Outro aspecto metodológico que pode ser analisado foi a técnica utilizada para a coleta de dados. No Gráfico 2, é possível observar quais as técnicas mais utilizadas nas coletas. Pontuaseque muitos trabalhos adotaram mais de uma técnica, por isso o somatório dos números dos dados ultrapassa os 64 artigos analisados. 
Gráfico2: Técnica de coleta de dados utilizada.

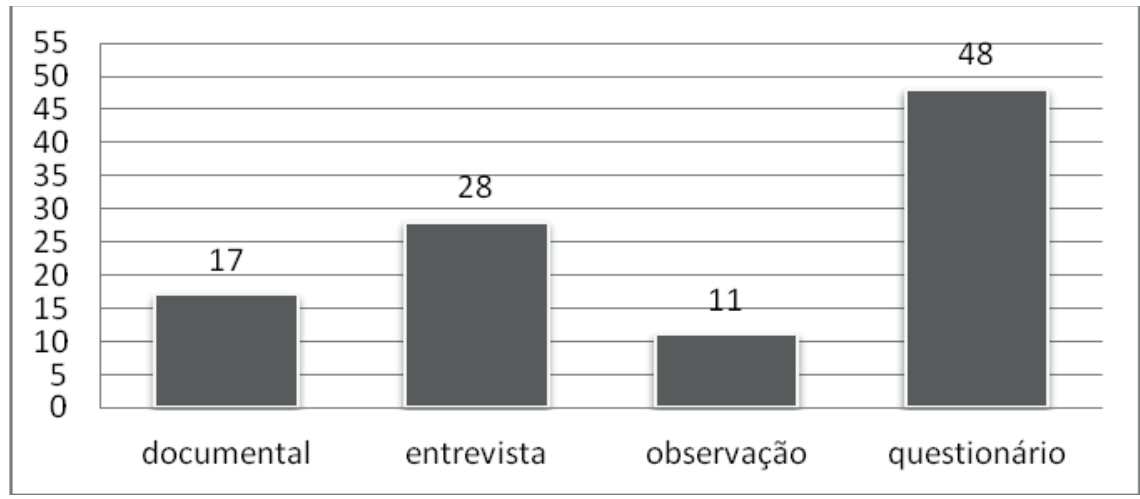

Fonte: Elaborado pelos autores.

É perceptível, na amostra pesquisada, a predominância do uso de questionários para a coleta dos dados. Tal predominância é motivada, certamente, pelo fato dos modelos teóricos de análise da QVT serem padronizados em forma de questionários, com diversas variáveis a serem analisadas, as quais se tornam mais práticas se verificadas por meio de questões fechadas com auxílio da escala Likert, do que em entrevistas.

Em relação ao número de autores por artigo, observa-se que é comum o trabalho em equipes pequenas, uma vez que artigos com um único autor ou com número elevado de autores não são predominantes, como mostra a Tabela 6.

Acredita-se que a opção por equipes concisas seja motivada pela dinâmica da pesquisa em si, pois, como o tema necessita de uma série de levantamentos in loco, dificulta o trabalho de um único pesquisador. Em contrapartida, a participação de muitos autores pode truncar o processo.

Tabela 6 - Produção de QVT por número de autores no artigo.

\begin{tabular}{ccccccccccccccccc}
\hline $\begin{array}{c}\text { Qtd autor/ } \\
\text { ano }\end{array}$ & $\mathbf{9 7}$ & $\mathbf{9 8}$ & $\mathbf{9 9}$ & $\mathbf{0 0}$ & $\mathbf{0 1}$ & $\mathbf{0 2}$ & $\mathbf{0 3}$ & $\mathbf{0 4}$ & $\mathbf{0 5}$ & $\mathbf{0 6}$ & $\mathbf{0 7}$ & $\mathbf{0 8}$ & $\mathbf{0 9}$ & $\mathbf{1 0}$ & $\mathbf{1 1}$ & TOTAL \\
\hline 1 & 0 & 1 & 1 & 1 & 1 & 1 & 1 & 1 & 0 & 0 & 1 & 0 & 1 & 0 & 0 & $\mathbf{9}$ \\
2 & 2 & 1 & 5 & 1 & 1 & 6 & 5 & 3 & 1 & 1 & 0 & 3 & 1 & 0 & 0 & $\mathbf{3 0}$ \\
3 & 0 & 0 & 1 & 1 & 1 & 2 & 1 & 0 & 3 & 1 & 0 & 2 & 0 & 0 & 0 & $\mathbf{1 2}$ \\
4 & 0 & 0 & 0 & 0 & 0 & 2 & 0 & 0 & 0 & 0 & 1 & 1 & 1 & 2 & 2 & $\mathbf{9}$ \\
5 & 0 & 0 & 0 & 0 & 0 & 0 & 0 & 0 & 2 & 0 & 0 & 1 & 0 & 0 & 0 & $\mathbf{3}$ \\
6 & 0 & 0 & 0 & 0 & 1 & 0 & 0 & 0 & 0 & 0 & 0 & 0 & 0 & 0 & 0 & $\mathbf{1}$ \\
TOTAL & $\mathbf{2}$ & $\mathbf{2}$ & $\mathbf{7}$ & $\mathbf{3}$ & $\mathbf{4}$ & $\mathbf{1 1}$ & $\mathbf{7}$ & $\mathbf{4}$ & $\mathbf{6}$ & $\mathbf{2}$ & $\mathbf{2}$ & $\mathbf{7}$ & 3 & 2 & $\mathbf{2}$ & $\mathbf{6 4}$ \\
\hline
\end{tabular}

Fonte: Elaborado pelos autores.

Em relação ao método utilizado, nota-se, no Gráfico 3, as técnicas mais utilizadas. Cabe pontuar que um artigo pode ter uma pesquisa de natureza múltipla. 
Gráfico3: Produção de QVT por tipo de análise de dados utilizada no artigo.

Análise Quantitativa

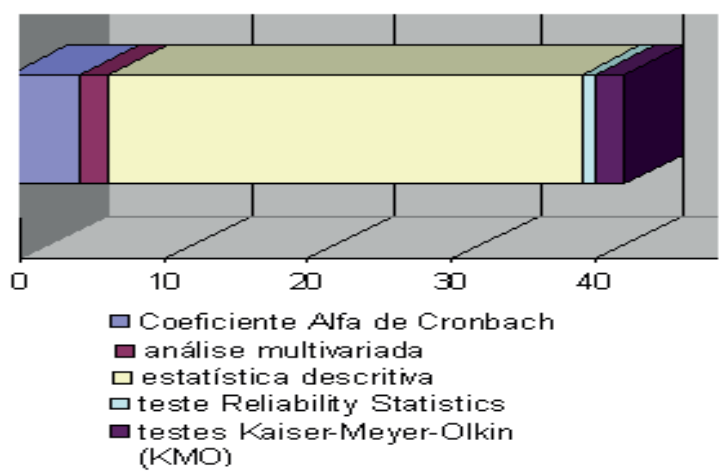

Análise Qualitativa

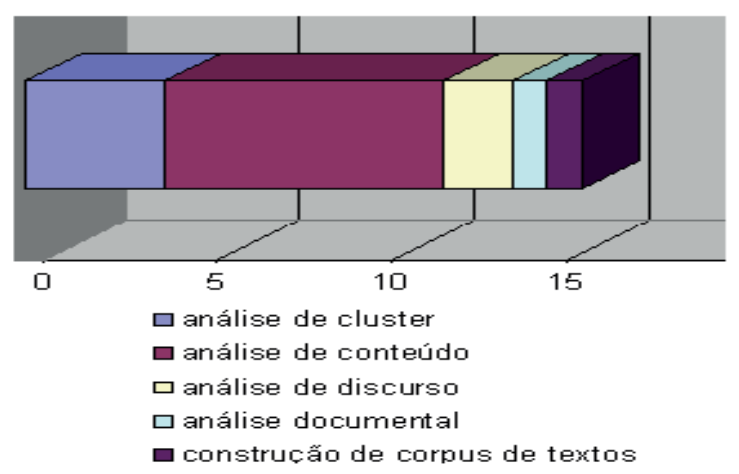

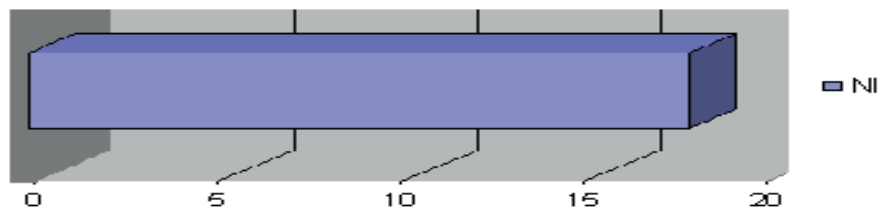

Fonte: Elaborado pelos autores.

Verifica-se que, nas análises quantitativas, existe a predominância do uso de estatística descritiva, já nas qualitativas é mais comum o uso de análise de conteúdo para efetuar a análise dos dados coletados.

Também foram investigados os modelos de análise de QVT e as referências mais utilizadas para elaboração das pesquisas, conforme a Tabela 7 e o Gráfico4.

Tabela 7: Modelos utilizados nos artigos analisados.

\begin{tabular}{lcccccccccccccccc}
\hline \multicolumn{1}{c}{$\begin{array}{c}\text { Modelo QVT/ } \\
\text { Período }\end{array}$} & $\mathbf{9 7}$ & $\mathbf{9 8}$ & $\mathbf{9 9}$ & $\mathbf{0 0}$ & $\mathbf{0 1}$ & $\mathbf{0 2}$ & $\mathbf{0 3}$ & $\mathbf{0 4}$ & $\mathbf{0 5}$ & $\mathbf{0 6}$ & $\mathbf{0 7}$ & $\mathbf{0 8}$ & $\mathbf{0 9}$ & $\mathbf{1 0}$ & $\mathbf{1 1}$ & TOTAL \\
\hline Cooper et. al. (1988) & 0 & 0 & 2 & 1 & 1 & 1 & 1 & 0 & 0 & 1 & 0 & 1 & 0 & 0 & 0 & $\mathbf{8}$ \\
Fernandes (1996) & 0 & 0 & 0 & 0 & 0 & 1 & 1 & 1 & 1 & 0 & 0 & 1 & 3 & 0 & 0 & $\mathbf{8}$ \\
Hackman e Oldham & 0 & 1 & 4 & 2 & 2 & 3 & 1 & 1 & 2 & 1 & 0 & 0 & 0 & 0 & 0 & $\mathbf{1 7}$ \\
(1975) & 1 & 1 & 3 & 0 & 1 & 2 & 3 & 1 & 2 & 0 & 0 & 1 & 3 & 2 & 1 & $\mathbf{2 1}$ \\
Walton (1973) & 1 & & & & 17 & & & \\
Werther e Davis & 0 & 0 & 1 & 0 & 0 & 0 & 1 & 0 & 1 & 0 & 0 & 0 & 2 & 2 & 0 & $\mathbf{7}$ \\
(1983) & 1 & 0 & 1 & 1 & 0 & 2 & 1 & 0 & 1 & 0 & 0 & 1 & 1 & 1 & 0 & $\mathbf{1 0}$ \\
Westley (1979) & 0 & 0 & 1 & 0 & 0 & 2 & 0 & 0 & 0 & 0 & 0 & 0 & 0 & 0 & 0 & $\mathbf{3}$ \\
Marques (1993) & 0 & 0 & 1 & 1 & 2 & 0 & 4 & 0 & 4 & 0 & 0 & 3 & 2 & 0 & 2 & $\mathbf{1 9}$ \\
Outros & 1 & 0 & 1 & 0 & 0 & 6 & 2 & 1 & 0 & 1 & 2 & 3 & 1 & 0 & 0 & $\mathbf{1 8}$ \\
MP/NI & $\mathbf{3}$ & $\mathbf{2}$ & $\mathbf{1 4}$ & $\mathbf{5}$ & $\mathbf{6}$ & $\mathbf{1 7}$ & $\mathbf{1 4}$ & $\mathbf{4}$ & $\mathbf{1 1}$ & $\mathbf{3}$ & $\mathbf{2}$ & $\mathbf{1 0}$ & $\mathbf{1 2}$ & $\mathbf{5}$ & $\mathbf{3}$ & $\mathbf{1 1 1}$ \\
\hline TOTAL & & & & & & & & & &
\end{tabular}

Fonte: Elaborado pelos autores

Como se pode observar, os artigos, em geral, baseiam-se em mais de um modelo. Percebese, porém, a predominância dos modelos de Hackman e Oldham (1975), Marques (1993) e de Walton (1973). Nota-se, também, que é significativo o número de artigos que fazem uso de modelos próprios - resultantes, geralmente, da integração de partes dos modelos anteriores e/ou que não relatam em qual modelo se basearam. Isso é corroborado pela análise das referências utilizadas nos artigos (Gráfico 4), em que Hackman e Oldham (1975) e Walton (1973) aparecem como os mais referenciados. 
Quadro4: Referências utilizadas nos artigos de QVT.

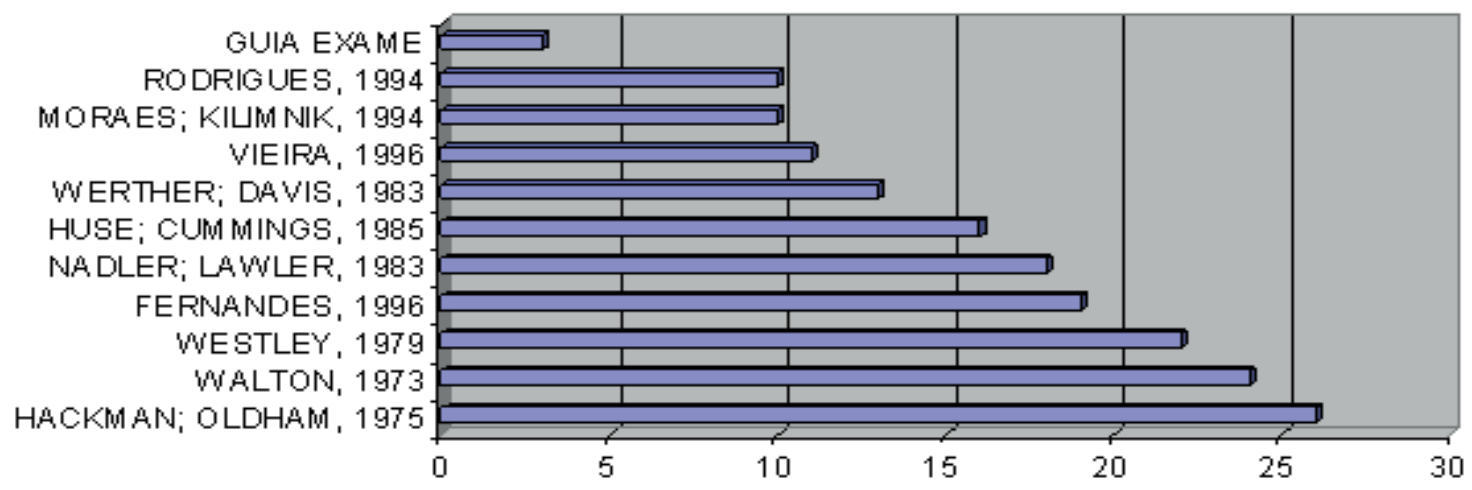

Fonte: Elaborado pelos autores.

Quanto à concepção e abordagem de QVT utilizada, percebe-se, dentre a amostra analisada, que a maioria dos artigos publicados nos anais do EnANPAD abordam a QVT como um instrumento de diagnóstico, existindo poucos estudos que enfocam o tema sob uma perspectiva que pode ser relacionada a outro tema ou aspecto organizacional, como desempenho ou responsabilidade social. O Quadro 1, abaixo, evidencia isso.

Quadro 1 - Utilização da QVT.

\begin{tabular}{|l|c|l|}
\hline \multicolumn{1}{|c|}{ Utilização da QVT } & $\begin{array}{c}\text { Qtd. } \\
\text { Artigos }\end{array}$ & \multicolumn{1}{c|}{ Exemplos } \\
\hline Diagnosticar QVT & 39 & $\begin{array}{l}\text { Um artigo utilizou o modelo de Hackman e Oldham (1975) } \\
\text { para diagnosticar o índice de QVT em uma microempresa } \\
\text { do comércio varejista. }\end{array}$ \\
\hline $\begin{array}{l}\text { Relacionar QVT com outro } \\
\text { tema }\end{array}$ & 10 & $\begin{array}{l}\text { Um artigo procurou relacionar a QVT com o controle exer- } \\
\text { cido sobre os empregados, outro buscou relacionar a QVT } \\
\text { com a responsabilidade social e outro buscou relacioná-la } \\
\text { com o nível de desempenho organizacional. }\end{array}$ \\
\hline $\begin{array}{l}\text { Diagnosticar QVT e relacioná } \\
\text {-la com outro tema }\end{array}$ & 15 & $\begin{array}{l}\text { Um artigo procurou identificar o nível de QVT em algumas } \\
\text { organizações privadas e verificar seus reflexos no compro- } \\
\text { metimento dos colaboradores. }\end{array}$ \\
\hline
\end{tabular}

Fonte: Elaborado pelos autores.

Em relação aos autores dos artigos, verificam-se questões relacionadas à instituição a que os autores estão vinculados e identificar os autores que mais publicam na área. No Gráfico 5, é apresentada a produção acadêmica por cada instituição. 
Gráfico5: Número de vezes em que cada instituição aparece vinculada ao autor do artigo.

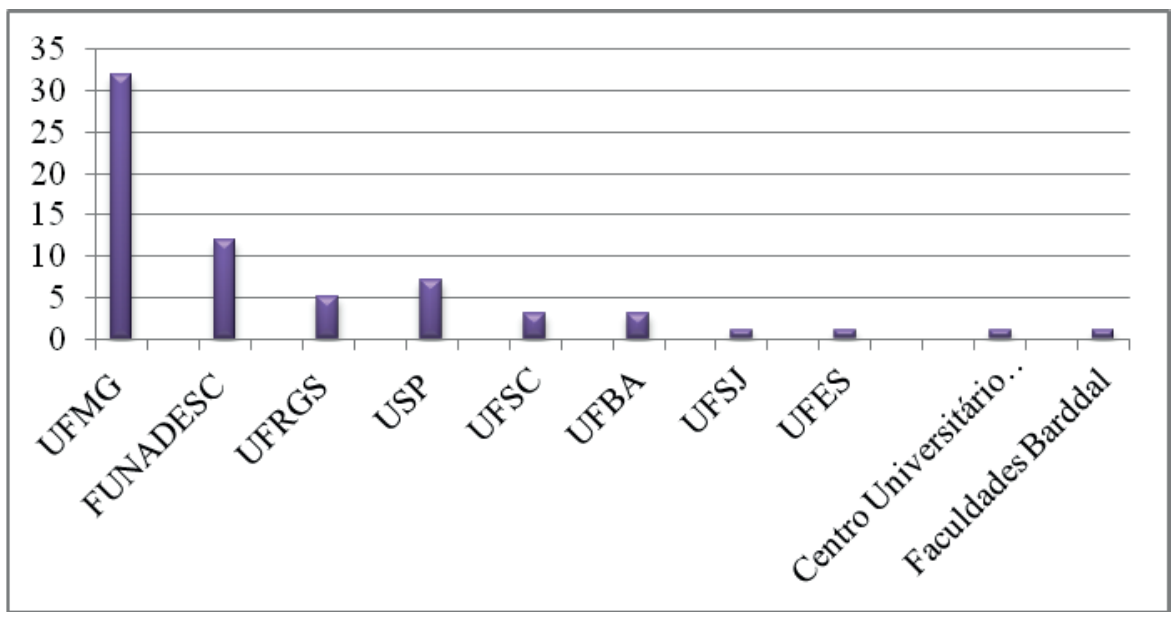

Fonte: Elaborado pelos autores.

Verifica-se no gráfico anterior que, dentre o escopo desta pesquisa, a Universidade Federal de Minas Gerais (UFMG) apresenta-se como referência na produção de pesquisas científicas sobre QVT, apresentando um número de autores maior do que o dobro do número de autores provenientes da Fundação Nacional de Desenvolvimento do Ensino Superior Particular (FUNADESP), segunda instituição que mais publica. Aparecem, ainda, outras instituições que publicaram apenas duas ou menos vezes nos artigos analisados.

Por fim, verificam-se quais autores publicaram mais de um artigo sobre QVT no evento pesquisado, conforme mostra a Tabela 8.

Tabela 8: Produção sobre QVT por autor.

\begin{tabular}{|c|c|c|c|c|c|c|c|c|c|c|c|c|c|c|c|c|}
\hline Autor / ano & 97 & 98 & 99 & 00 & 01 & 02 & 03 & 04 & 05 & 06 & 07 & 08 & 09 & 10 & 11 & $\begin{array}{l}\text { Qtd. } \\
\text { Artigos }\end{array}$ \\
\hline Lúcio Flávio Renault de Moraes & 1 & & 2 & 1 & 2 & & & 1 & & & & & & & & 7 \\
\hline Antonio Luiz Marques & & & 2 & 1 & 1 & & & 1 & 1 & & & & & 1 & & 7 \\
\hline Luiz Carlos Honório & & & 1 & 1 & 1 & & & & & & & 1 & & & & 4 \\
\hline Maria Auxiliadora Diniz de Sá & & & 1 & & & & & & 1 & & 1 & 1 & & & & 4 \\
\hline Ana Cristina Limongi França & 1 & & & & & 1 & & & & & & 1 & 1 & & & 4 \\
\hline Valmíria Carolina Piccinini & & 1 & & & & 2 & & & & & & & & & & 3 \\
\hline $\begin{array}{l}\text { Washington Jose } \\
\text { de Souza }\end{array}$ & & & & & & & 2 & & 1 & & & & & & & 3 \\
\hline Zelia Miranda Kilimnik & & & 1 & & & 1 & & & 1 & & & & & & & 3 \\
\hline Anderson de Souza Sant'anna & & & 1 & & & & & & & 1 & & & & & & 2 \\
\hline Daniela Cristina Guimarães & & & & & & & 1 & 1 & & & & & & & & 2 \\
\hline José Bezerra Honório & & & & & & & & & & & 1 & 1 & & & & 2 \\
\hline Karoline Morais Porto Viana & & & & & & & & & & & 1 & 1 & & & & 2 \\
\hline Kely César Martins de Paiva & & & 1 & & & 1 & & & & & & & & & & 2 \\
\hline Rodrigo Cesar Reis de Oliveira & & & & & & & & & & & 1 & 1 & 1 & & & 3 \\
\hline Suzana da Rosa Tolfo & & 1 & & & & 1 & & & & & & & & & & 2 \\
\hline Vania Medianeira Flores Costa & & & & & & & 1 & & & 1 & & & & & & 2 \\
\hline Outros & 2 & 1 & 5 & 3 & 8 & 21 & 10 & 4 & 17 & 3 & 1 & 15 & 5 & 7 & 8 & - \\
\hline
\end{tabular}

Fonte: Elaborado pelos autores. 
Na Tabela 8, verifica-se que dois autores se destacam na produção científica sobre o a QVT e os temas a ela relacionados. Lúcio Flávio Renault de Moraes destaca-se na produção científica por pesquisar sobre os seguintes temas: QVT, comprometimento e comportamento organizacional, e Antonio Luiz Marques por pesquisar diversos temas acerca da área de recursos humanos. Ressalta-se, ainda, que a autora Ana Cristina Limongi França, embora seja uma das autoras mais citadas sobre o tema, publicou no EnANPAD apenas 4 trabalhos, o que pode significar que trabalhos mais maduros não estejam sendo apresentados neste evento, pois os autores estão buscando publicar em eventos específicos da área de Gestão de Pessoas, como o Encontro da Divisão Acadêmica de Gestão de Pessoas e Relações de Trabalho (EnGPR) ou em eventos internacionais.

\section{CONSIDERAÇÕES FINAIS}

Percebe-se que a qualidade de vida no trabalho (QVT) é um tema que vem sendo posto em pauta pelas organizações. Isso deve-se ao fato do tema estar intimamente ligado às pessoas, afetando diretamente a produtividade do corpo funcional, e consequentemente, o desempenho geral da organização.

Ao abordar o tema da QVT, o presente artigo, na forma de estudo bibliométrico, procurou caracterizar e analisar a produção científica referente ao Encontro da Associação Nacional de PósGraduação e Pesquisa em Administração (EnANPAD), no período de 1997 a 2011.

Porém, apesar da importância das pessoas nas organizações ser constantemente abordada em discursos da prática empresarial, nas pesquisas científicas o tema ainda é abordado de forma incipiente. Já em relação aos objetivos, as pesquisas acontecem muito mais com um cunho investigativo para um diagnóstico do índice de QVT, que com o intuito de verificar seus reflexos em todo o contexto organizacional. Essa afirmação remete ao seguinte questionamento: até que ponto a QVT vem sendo abordada nas organizações por consciência da sua importância e participação no desempenho das estratégias organizacionais, ou por um mero modismo, por uma forma de controle ou por um "benefício" aos empregados?

Sendo assim, como recomendações para pesquisas futuras, sugere-se que se verifique a relação da QVT com demais os aspectos organizacionais, buscando compreender até que ponto a alta gerência das organizações veem a QVT como uma estratégia organizacional e não como um simples instrumento de diagnóstico. 


\section{REFERÊNCIAS}

ALBUQUERQUE, L. G.; LIMONGI-FRANÇA, A. C. Estratégias de Gestão de Pessoas e gestão da qualidade de vida no trabalho: o stress e a expansão do conceito de qualidade total. Revista de Administração, São Paulo, v. 33, n. 2, p. 40-51, Abr./Jun. 1998.

BARRETO, L. M. T. da S.; SILVA, M. P. da; FISCHER, A. L.; ALBUQUERQUE, L. G. de; AMORIM, W. A. C. de. Temas Emergentes em Gestão de Pessoas: Uma Análise Da Produção Acadêmica. Revista de Administração da UFSM, Santa Maria, v. 4, n.1, p. 215-232, mai./ ago. 2011.

BERTERO, C. O.; CALDAS, M. P.; WOOD JR, T. Produção Científica em administração de Empresas: Provocações, Insinuações e Contribuições para um Debate Local. Revista de Administração Contemporânea, Curitiba, v. 3, n. 1, p. 147-178, jan./abr. 1999.

BERTERO, C. O.; CALDAS, M. P.; WOOD JR, T. Produção Científica em Administração de Empresas: Provocações, Insinuações e Contribuições para um Debate Local. In: Encontro Da Associação Nacional De Programas De Pós-Graduação Em Administração. Anais... Foz do Iguaçu: ANPAD, 1998.

CAPPELLE, M. C. A.; BRITO, M. J.; MELO, M. C. O. L.; VASCONCELOS, K. A. A Produção Científica sobre Gênero na Administração: Uma Meta-Análise. In: Encontro da Associação Nacional de Programas de Pós-Graduação em Administração. Anais... Salvador: ANPAD, 2006.

CARDOSO, W. L. C. D. Qualidade de Vida e Trabalho: uma articulação possível. São Paulo: Casa do Psicólogo, 1999.

CLOSS, L. Q.; ARAMBURÚ, J. V.; ANTUNES, E. D. Produção Científica sobre o Ensino em Administração: uma Avaliação Envolvendo o Enfoque do Paradigma da Complexidade. In:
Encontro da Associação Nacional de Programas de Pós-Graduação em Administração. Anais... Salvador: ANPAD, 2006.

COOPER, C.L.; SLOAN, S.J.; WILLIAMS, S. Occupational stress indicator management guide. Windsor: NFER-Nelson, 1988. 102p.

DANTAS, R.S.A.; SAWADA, N.O., MALERBO, M.B. Pesquisas sobre qualidade de vida: revisão da produção científica das Universidades Públicas do Estado de São Paulo. Revista Latino Americana de Enfermagem, v. 11, n. 4, p.532-538, 2003.

FERNANDES, E. Qualidade de Vida no Trabalho: como medir para melhorar. Salvador: Casa da Qualidade, 1996.

FLEURY, S. (Org.). Análise do Perfil dos Artigos Publicados na Revista de Administração Pública - RAP - No Período 1992-2002. Rio de Janeiro: EAESP/FGV, 2003.

FRIEDMAN, T. L. O Mundo é Plano: Uma Breve História do Século XXI. Trad. Cristiana Serra, S. Duarte. Rio de Janeiro: Objetiva, 2005.

GHENO, R.; BERLITZ, J. Remuneração Estratégica e Pacote de Benefícios: Um Estudo de Caso Aplicado ao Nível Operacional de Uma Multinacional. Revista de Administração da UFSM, Santa Maria, v. 4, n.1, p. 268-287 mai./ ago. 2011.

HACKMAN, J. R.; OLDHAM, G. R. Developmentofthejobdiagnosticsurvey. Journal of Applied Psychology, v. 60, n. 2, p. 159-170, 1975.

HUSE, E.; CUMMINGS, T. Organization development and change. St. Paul: West, 1985.

JARDIM, R.; BARRETO, S. M., ASSUNÇÃO, A. A.. Condições de trabalho, qualidade de vida e disfonia entre docentes. Caderno Saúde Pública, Rio de Janeiro, v. 23, n.10, p. 24392461, out, 2007. 
KUHNEN, V. J.; KESTRING, S. Teoria e Prática da Metodologia científica. Blumenau, Nova Letra, 2004.

LIMONGI-FRANÇA, Ana Cristina. Qualidade de vida no trabalho - conceitos e práticas nas empresas da sociedade pós-industrial. São Paulo: Atlas, 2003.

MACHADO-DA-SILVA, C. L.; CUNHA, V. C.; AMBONI, N. Organizações: O estado da arte da produção acadêmica no Brasil. In: Encontro da Associação Nacional de Programas de PósGraduação em Administração. Anais... Belo Horizonte: ANPAD, 1990.

MARQUES, A. L. The work situation and class position of brazilian engineers. England: The University of Aston in Birmingham, 1993.

MEDEIROS, J. P.; OLIVEIRA, J. A. Uma Viagem à Produção Científica em Qualidade de Vida no Trabalho (QVT) nos anos 2001 a 2005: Estudo nos Anais do ENANPAD. In: I Encontro de Gestão de Pessoas e Relações de Trabalho. Anais... Natal: ANPAD, 2007.

MENDES, N. C. N; OLIVEIRA, J. A. Qualidade de Vida no Trabalho dos profissionais de Saúde: Um estudo dos enfermeiros de nível superior de dois hospitais de Natal-RN. Série cadernos de estudos do GERHQUAL - No. 02. Natal: UFRN, 1998, 41p. [relatório de pesquisa]. (www.ufrn.br/gerhqual).

NADLER, D.; LAWLER, E. Quality of work life: perspectives and directions. Organization Dynamics, v. 1, n. 11, p. 20-30, Winter, 1983.

OMS. Organização Mundial da Saúde. Classificação internacional de funcionalidade, incapacidade e saúde. São Paulo: Edusp; 2003.

POT, F. Productivity and utilisation of the potential workforce. Texto apresentado na disciplina Qualidade de Vida no trabalho, FEA/ USP, 2 o trimestre de 2003.

RODRIGUES, M. V. C. Qualidade de Vida no Trabalho: evolução e análise no nível gerencial.
Petrópolis: Editora Vozes, 1999.

ROSA, M. dos S.; IBDAIWI, T.K. Fatores Responsáveis Pelo Turnover Em Uma Empresa de

Fast Food de Santa Maria - RS. Revista de Administração da UFSM, Santa Maria, v. 5, n. 3, p. 525-538, set./dez. 2012

ROSSONI, L.; FERREIRA JÚNIOR, I.; HOCAYENDA-SILVA, A. J. Administração de Ciência e Tecnologia: A Produção Científica Brasileira entre 2000 e 2005. In: Simpósio De Gestão Da Inovação Tecnológica. Anais... Gramado: ANPAD, 2006.

SAMPAIO, Jader dos Reis e colaboradores. Qualidade de vida no trabalho e psicologia social. 2.ed. São Paulo: Casa do Psicólogo, 2004.

SCHNEIDER, A.B.; CARNEIRO, M. L.; DUTRA, A. Análise da Produção Científica Brasileira sobre Avaliação de Desempenho Organizacional no Período de 1997 a 2007. In: IV Encontro de Estudos em Estratégia / 3Es. Anais... Recife: ANPAD, 2009.

SERRA, F. A. R.; FERREIRA, M. ; PEREIRA, M. F. ; LISSONI, J. Evolução da pesquisa em rbv: um estudo dos últimos EnANPAD's. Revista Brasileira de Estratégia, v. 1, p. 39-56, 2008.

TOLFO, S. R.; PICCININI, V.C. As melhores empresas para trabalhar no Brasil e a Qualidade de Vida no Trabalho: Disjunções entre a teoria e a prática. Revista de Administração Contemporânea. Curitiba, V.5, n.1, p.165-193, jan/abril. 2001

WALTON, R. E. Quality of working life: what is it? Sloan Management Review, v. 15, n. 1, p. 11-21, Dec. 1973.

WERTHER, W; DAVIS, K. Administração de pessoal e recursos humanos. São Paulo: McGraw-Hill, 1983.

WESTLEY, W. A. Problems and solutions in the 
O RECURSO MAIS IMPORTANTE PARA AS ORGANIZACÕES SÃO MESMO AS PESSOAS? UMA ANÁLISE DA PRODUÇÃO CIENTIIFICA SOBRE QỦALIDADE DE VIDA NO TRABALHO (QVT)

quality of working life. Human Relations, v. 32, n. 2, p. 111-123, Feb. 1979.

WOOD JR, T.; PAULA, A. P. P. Top-management: pesquisa sobre as revistas populares de gestão no Brasil. In: Encontro Da Associação Nacional De Programas De Pós-Graduação Em Administração. Anais... Salvador: ANPAD, 2002. 